\title{
Philosophiques
}

\section{Avortement Oui/Non, propos recueillis par Michel Buruiana, Québec, Humanitas, 1988, 120 pages.}

\section{Jocelyne Saint-Arnaud}

Volume 17, numéro 2, automne 1990

URI : https://id.erudit.org/iderudit/027136ar

DOI : https://doi.org/10.7202/027136ar

Aller au sommaire du numéro

Éditeur(s)

Société de philosophie du Québec

ISSN

0316-2923 (imprimé)

1492-1391 (numérique)

Découvrir la revue

Citer ce compte rendu

Saint-Arnaud, J. (1990). Compte rendu de [Avortement Oui/Non, propos recueillis par Michel Buruiana, Québec, Humanitas, 1988, 120 pages.]

Philosophiques, 17(2), 229-232. https://doi.org/10.7202/027136ar d'utilisation que vous pouvez consulter en ligne.

https://apropos.erudit.org/fr/usagers/politique-dutilisation/ 
Avortement Out/Non, propos recueillis par MICHEL BURUIANA, Québec, Humanitas, 1988, 120 pages.

par Jocelyne Saint-Arnaud

Suivant la pratique américaine, l'avortement, en contexte canadien, est une question qui est d'abord débattue devant les tribunaux. En l'absence d'une législation en cette matière, les juges s'appuient en général sur les chartes des droits pour solutionner un problème qui est essentiellement éthique et politique : doit-on permettre le libre accès à l'avortement, doit-on l'interdire ou encore doit-on le réglementer?

Dans un tel contexte, il est heureux que le livre de Michel Buruiana, Avortement oui/non, porte le débat sur la place publique. Ce livre se présente comme un * dialogue parallèle * entre deux tenants de positions contraires face à l'avortement : le docteur Morgentaler qui lutte depuis une dizaine d'années pour maintenir ouvertes ses cliniques d'avortement et Mgr Blanchet, évêque de Gaspé et biologiste. Chacun y déve- 
loppe une argumentation qui met en lumière différents aspects de la question.

Henry Morgentaler se présente lui-même comme un humaniste; son argumentation se situe au niveau du normatif et repose sur quelques principes fondamentaux. En bref, il faut éviter aux femmes la souffrance et le danger des avortements clandestins, la femme doit avoir le droit d'accepter ou de refuser le foetus, car c'est en faisant des choix qu'elle exerce pleinement son humanité; enfin, comme le bien-être de la société passe par le bien-être individuel, plus les femmes pourront exercer leur libre choix, mieux la société s'en portera.

Bertrand Blanchet utilise une argumentation plus diversifiée et s'inspire de la multidimensionnalité du problème pour élargir la perspective du débat à l'échelle de la société. Mal à l'aise de se prononcer sur un sujet qui concerne d'abord les femmes, il reconnaît que la femme porte pratiquement seule le fardeau de la grossesse et même celui de la naissance. Cependant, il milite en faveur du respect de la vie à partir de la base ontologique suivante: le développement de l'embryon ne peut se catégoriser par étapes significatives en regard d'un avortement possible, puisque, dès la fécondation, il y a la présence d'un individu dont le développement est coordonné de façon continue. Comme il est impossible de fixer des seuils de complexité de vie qui ne sont pas arbitraires, l'avortement ne doit pas être permis même durant les premiers mois de la grossesse. Mieux vaut s'attaquer à la racine du problème et examiner quelles sont les raisons profondes pour lesquelles les couples refusent des enfants. ${ }^{1}$ Selon Mgr Blanchet, c'est toute la société qui doit se remettre en question.

N'étant pas un théoricien, Henry Morgentaler apporte des arguments qui sont sans aucun doute moins bien étayés que ceux de son opposant. Il n'en demeure pas moins que la liberté a été reconnue comme un droit fondamental autant par les philosophes qui fondent les droits sur un état de nature, de John Locke à Robert Nozick, que par ceux qui les fondent en raison, d'Emmanuel Kant à John Rawls. H.L.A. Hart pose l'existence hypothétique des droits fondamentaux de la façon suivante: «s'il existe des droits fondamentaux, il s'ensuit qu'il y a au moins un droit naturel, le droit égal pour tous les êtres d'être libres *², ce qui signifie que tout individu capable de choix a le droit d'agir sans subir la coercition et la contrainte des autres envers lui et, corrélativement, il possède la liberté d'action en autant qu'il n'exerce pas de coercition ou de contraintes sur les autres. Le problème avec la théorie des droits, c'est qu'elle résoud le problème de l'avortement en autant qu'aucun droit n'est conféré à l'embryon. Autrement dit, la femme

1. Monique Dumais dans un chapitre du livre De l'étbique a la bioéthique : repères en soins infirmiers, (Chicoutimi, Gaëtan Morin, 1986) intitule "l'avortement *, cite le Conseil des affaires sociales et de la famille du Québec pour indiquer que les motifs allégués sont d'ordre médical, mais aussi d'ordre familial, social et économique. Il semblerait meme que les « motifs les plus fréquemment évoqués pour obtenir un avortement seraient surtout d'ordre social et économique. Ce sont: le nombre déja suffisant d'enfants, l'age de l'enfant précédent, l'age avancé de la mère, une situation financière plutôt difficile, l'effet sur le travail.

2. H.L.A. Hart, *Are There Any Natural Rights*, The Pbilosopbical Review, vol. 64, $\mathrm{n}^{\circ} 2$ (1955), p. 189 
pourrait librement avoir recours à l'avortement, sauf si des droits sont reconnus au fotus. Il est évident que le fœtus n'a pas la capacité de faire des choix, mais la loi ne doit-elle pas le protéger au même titre que les incapables? Mais pourquoi protéger le foetus?

Bertrand Blanchet répondrait que le foetus est déjà un individu qui contrôle son développement d'où la nécessité d'éviter l'avortement. Cependant, d'autres théoriciens s'appuyant aussi sur des faits biologiques pourraient soutenir que l'autonomie du fortus ne se manifeste vraiment qu'à la dixième semaine et qu'en-deçà de cette période un avortement peut en toute moralité être pratiqué. En fait, la preuve ontologique ne fera jamais l'unanimité. Poser la question du développement autonome du fœtus, c'est comme se demander à partir de quel moment, s'il en est, un embryon peut être considéré comme un être humain à part entière et avoir des droits juridiques reconnus. Pour le code criminel, un être humain n'a des droits qu'à partir du moment où il est né et pas avant, d'où la difficulté pour la Cour actuellement de protéger le foetus ou l'embryon quel que soit son étape de développement.

Pourtant dans la perspective bioéthique actuelle, la question des droits de l'embryon se pose différemment. Non seulement l'embryon peut vivre de plus en plus tôt hors du ventre de la mère, ce qui repousse continuellement les limites de l'avortement si le critère de viabilité est déterminant, mais encore il n'est pas inconcevable qu'un embryon conçu in vitro puisse se développer complètement en dehors du ventre de la mère dans un milieu entièrement artificiel créé par la science et la technologie biomédicales. Peut-être ne s'agit-il que d'une utopie ? Cependant, la science moderne rêve de maîtriser de nouveaux horizons par l'expérimentation sur l'embryon humain : congélation, clonage et manipulation génétique ne représentent que quelques possibilités offertes. En dehors du débat ontologique portant sur les faits biologiques significatifs pour résoudre la question de l'avortement, il existe de fortes raisons bioéthiques pour conférer des droits à l'embryon. Ces raisons sont de l'ordre de l'intérêt général et de l'avenir de la société, car les nouvelles technologies de la reproduction représentent, sous de nombreux aspects, des menaces pour l'avenir du genre humain. Chose certaine, elles ont le pouvoir de changer complètement nos rapports à la reproduction, de même que les structures économiques et sociales qui se fondent sur nos rapports à la reproduction. L'unanimité pourrait donc être faite sur la nécessité de conférer des droits aux foctus et à l'embryon, droits qui protégeraient les fœtus et les embryons, et qui permettraient d'éviter un contrôle scientifique de l'avenir de la race humaine.

Si l'embryon a des droits, fondés ontologiquement sur le développement embryonnaire ou reconnus comme étant une question d'intérêt général, alors la liberté de la mère s'arrête où commence celle de l'enfant à naître.

Respecter le libre choix, c'est accepter que, dans un même hôpital, coexistent des cliniques de fertilité et des cliniques d'avortement, que l'avortement soit un moyen contraceptif défrayé par l'État au même titre que les primes à la maternité et les allocations familiales. Cependant, au-delà des problèmes de la distribution des soins, il y a le drame humain 
auquel fait référence Henry Morgentaler de la mère qui refuse l'enfant et surtout, ce qui est philosophiquement plus significatif, des raisons pour lesquelles une mère refuse l'enfant, ce sur quoi se penche Bertrand Blanchet. Ce dernier point mérite qu'on s'y attarde. La société égalitaire au niveau des relations hommes/femmes n'existe pas et il arrive trop souvent que la femme porte seule le poids et la responsabilité des enfants. Dans cette perspective, comment imposer à une autre personne un fardeau qu'elle sera seule à assumer? L'infanticide, dont le pourcentage est à la hausse, est-il préférable à l'avortement ? L'avortement n'est cependant pas la seule solution à ce problème. L'adoption ne permettait-elle pas autrefois une solution plus rationnelle, moins coûteuse en énergies et en techniques que les choix proposés par les sciences médicales contemporaines?

Entre les deux extrêmes, c'est-à-dire entre l'acceptation et le rejet inconditionnels de toutes les grossesses, il y a une position intermédiaire qui n'est pas abordée dans le livre. Cette position doit tenir compte des droits de la mère et de ceux du fœtus, de la liberté individuelle et de l'intérêt général de la société. La question de l'avortement déborde maintenant le cadre de la morale, entendue au sens traditionnel du terme. Les nouvelles technologies reliées aux origines de la vie nous forcent à établir des limites à ce qui est possible, de manière à protéger les foetus, mais aussi les femmes elles-mêmes de façon à ce qu'elles ne soient pas les victimes du progrès des sciences et des technologies biomédicales.

Université de Montréal. 